PHYSICAL REVIEW E 71, 011405 (2005)

\title{
Topological lifetimes of polydisperse colloidal hard spheres at a wall
}

\author{
Roel P. A. Dullens and Willem K. Kegel \\ Van 't Hoff Laboratory for Physical and Colloid Chemistry, Debye Institute, Utrecht University, Padualaan 8, 3584 CH Utrecht, \\ The Netherlands \\ (Received 7 September 2004; published 25 January 2005)
}

\begin{abstract}
Confocal scanning laser microscopy was used to study the behavior of dense suspensions of model colloidal hard spheres at a single wall. Due to the slight polydispersity, our system shows a reentrant melting transition at high densities involving a hexatic structure [R. P. A. Dullens and W. K. Kegel, Phys. Rev. Lett 92, 195702 (2004)]. The reentrant melting transition is accompanied by an increase in the mean-squared displacement. The correlation between structure and dynamics was quantitatively analyzed on a single-particle level. In particular, the topological lifetime, being the average time that a particle spends having the same coordination number, is determined for all coordination numbers and as a function of volume fraction. The defective (non-sixfoldcoordinated) particles exhibit shorter lifetimes than sixfold-coordinated particles, indicating that the mobility of the system is larger at or close to defective particles. The lifetime itself is a strong function of volume fraction. In particular, the global behavior of the mean-squared displacement is proportional to the hopping frequency (the inverse of the lifetime), showing that particles changing their coordination number contribute most to the local mobility.
\end{abstract}

DOI: 10.1103/PhysRevE.71.011405

PACS number(s): 82.70.Dd, 64.60.Cn, 64.70.-p

\section{INTRODUCTION}

The presence of walls can have an enormous impact on the behavior of a wide diversity of systems [1]. Also in colloid physics, the influence of walls has gained growing interest during the last decades [2]. In particular, the crystallization of colloids in confinement and at a single wall has been a continuous matter of interest [3-14]. For example, the existence of a prefreezing transition of hard spheres [8-11] and the enormous increase of the crystal nucleation rate in the vicinity of walls $[10,15]$ illustrate the remarkable impact of confinement. Prefreezing has also been reported in binary hard-sphere systems and colloid-polymer mixtures at a wall [16-19]. The perturbation of bulk behavior is even more pronounced if the bounding surface is patterned [6,20-22]. Furthermore, in completely confined two-dimensional (2D) systems a hexatic phase may appear as proposed in the Kosterlitz-Thouless-Halperin-Nelson-Young (KTHNY) theory for 2D crystal melting [23-25]. Due to the presence of free dislocations, the hexatic phase is characterized by shortrange translational order and quasi-long-range orientational order. Although several experiments [5,26-29] have validated the KTHNY melting scenario, the scenario has not been fully pinned down, in particular for hard disks.

Dynamical properties of confined (quasi-)2D colloidal systems have been investigated in many previous studies [5,30-39]. They demonstrated the presence of dynamical heterogeneities, which were also observed in $3 \mathrm{D}$ colloidal systems $[40,41]$. Dynamical heterogeneities arise as particles in given regions exhibit larger mobility than other regions. These particles typically exhibit cooperative stringlike motion [42]. As a consequence, the probability distribution of displacements shows strong devations from a single Gaussian distribution [40-42]. It was even suggested that multiple dynamical subsets of particles are present in dynamical het- erogeneous systems [40]. Furthermore, the existence of dynamical subsets might be related to structural differences between the subsets as there are clear indications that mobile particles tend to be in regions of higher disorder $[5,6,30,32,34-36,43]$.

Although the coupling between the structure and dynamics has been observed in several studies, there is hardly any direct quantitative measure of the structure-mobility relation. Weeks and Weitz showed that particles with larger displacement have fewer ordered neighbors [43]. Recently, it was proposed to examine dynamical heterogeneities in terms of fluctuations in the topological defect density [34]. These results point towards the importance of the defect structure with respect to the dynamics of the system. Here, we analyze on a single-particle level the correlation between the local structure and dynamics of slightly polydisperse hard spheres at a wall. Rather than considering (metastable) fluids, we also inspect this coupling throughout the whole range of structures observed in our system at the wall [7]. First of all, we characterize the defect structure as a function of the volume fraction $\phi$ quantitatively. Then, we correlate defect statistics to dynamics by analyzing the single-particle topology as a function of time for different coordination numbers. Subsequently, we define a topological lifetime, which characterizes the correlation between the local structure and dynamics. Finally, we discuss the behavior of the mean-squared displacement in terms of lifetimes and defects.

The paper is organized as follows. In Sec. II, we describe the colloidal model particles as well as the confocal microscopy setup. The data analysis (defect statistics, mean-squared displacement, and topological lifetimes) is explained in Sec. III. In Sec. IV first the (defect) structure of our system (Sec. IV A) is presented. Furthermore, the dynamics (Sec. IV B) and the relation between structure and dynamics in terms of topological lifetimes (Sec. IV C) are discussed. 


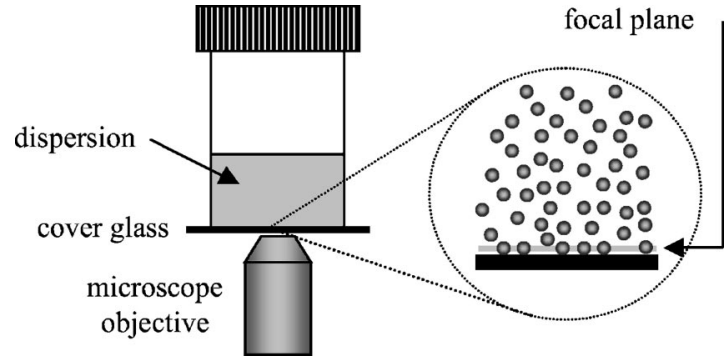

FIG. 1. Experimental setup (not to scale). The position of the focal plane is indicated.

\section{EXPERIMENT}

\section{A. Colloidal model system}

We used colloidal model hard spheres that could be imaged directly using confocal scanning laser microscopy. The particles consist of a core of silica, $450 \mathrm{~nm}$ in diameter, labeled with the fluorescent dye fluorescein isothiocyanate [44]. The cores are covered with a shell of nonfluorescent polymethylmethacrylate (PMMA) [45]. The system is sterically stabilized by a layer of 12-poly-hydroxystearic acid that is covalently linked to the PMMA [46]. The resulting silica-core-PMMA-shell particles were dispersed in a mixture of tetralin (Merck, for synthesis), cis-decalin (Merck, for synthesis), and carbon tetrachloride (Merck, for spectroscopy) which (nearly) matches the refractive index and the mass density of the colloids. The volume ratios were $30 \%$ tetralin, 30\% cis-decalin, and $40 \%$ carbon tetrachloride. In this mixture the particles behave as hard spheres [40]. The particle diameter $d$ is $1.4 \mu \mathrm{m}$ and the size polydispersity is $6 \%$ as estimated from scanning electron microscopy.

\section{B. Microscopy}

A Leica DM IRB confocal scanning laser microscope in combination with an argon laser $\left(\lambda_{0}=488 \mathrm{~nm}\right)$, a Leica TCS NT scanhead, and an oil-immersion lens (Leica $100 \times$, NA 1.4) was used in fluorescence mode. Using confocal microscopy, thin 2D cross sections of the sample are imaged. The thickness of this so-called focal plane is about $600 \mathrm{~nm}$ [47]. The colloidal suspension was contained in a small vial (contents $\sim 1 \mathrm{ml}$ ) [47]. The bottom of the vial was removed and replaced by a microscope cover glass (Chance Propper LTd., West Mids, England, $0.11 \mathrm{~mm}$ thickness), which was glued to the vial using an epoxy glue (Araldit AW2101 with hardener HW2951) (see Fig. 1). In this work, we imaged the particles present in the first layer at the glass wall, parallel to the flat glass bottom of the container. Hence, the $z$ position of the focal plane coincides with the particles present in the first layer at the glass wall (see Fig. 1). No signs of attractions between the particles and glass wall were found (as expected since the refractive index of the glass is matched by the solvent mixture).

The volume fraction $\phi$ of the samples was defined relative to the volume fraction at random close packing of $6 \%$ polydisperse hard spheres. This fraction was set at 0.66 [48]. By subsequently diluting the dispersion in the sample cell, the volume fraction was decreased from $\phi=0.64$ to $\phi=0.44$ in steps of typically 0.03. During the experiments the sample cell containing the dispersion was carefully weighed to correct for possible evaporation of the solvent. After each dilution step, the sample was thoroughly tumbled until the dispersion was fully homogenized (checked by confocal microscopy). At every volume fraction, time series of typically 100 images of the particles present in the first layer at the bottom wall were taken.

Of course, the wall-based layer is in contact with the bulk, which allows interlayer particle hopping. To make a 2D analysis of the first layer possible, interlayer hopping should be insignificant, which is the case in our experiment. First of all, at these volume fractions the system is strongly layered at the flat wall [3]. Furthermore, the particle displacements are very small compared to the interlayer distance (of the order of a particle diameter) as we will show later. Moreover, typically more than $90 \%$ of the particles are tracked throughout the whole time series, indicating that only an insignificant number of particles is lost. We also checked that at all volume fractions the number of particles is constant during the experiment. Thus, interlayer hopping is not expected to interfere with our 2D analysis of the wall baser layer.

\section{DATA ANALYSIS}

\section{A. Defect statistics}

Since the particles have a fluorescent core and a nonfluorescent shell, the particle centers could be easily retrieved with high accuracy from the raw microscopy images using image analysis software similar to those described in [49]. Correlating the particle positions within single frames using a Delaunay triangulation, the number of nearest neighbors of every particle (i.e., the coordination number $n_{c}$ ) was identified. The fraction of $n_{c}$-coordinated particles is then easily calculated:

$$
X_{n_{c}}=\frac{N_{n_{c}}}{N},
$$

where $N_{n_{c}}$ is the number of particles with coordination number $n_{c}$ and $N$ is the total number of particles.

\section{B. Mean-squared displacements}

Tracking the particles in time allows the analysis of the single-particle dynamics. First of all, we calculated the selfpart of the Van Hove correlation function $G_{s}$, being the probability distribution that a particle has traveled a distance $r$ in a time interval $t$ :

$$
G_{s}(r, t)=\frac{1}{N}\left\langle\sum_{i=1}^{N} \delta\left(r+r_{i}(0)-r_{i}(t)\right)\right\rangle .
$$

Subsequently, the mobility of the particles was measured in terms of the mean-squared displacement $\left\langle r^{2}\right\rangle$, which is defined as the second moment of $G_{s}$ :

$$
\left\langle r^{2}(t)\right\rangle=\sum_{i=1}^{N} r^{2}(t) G_{s}(r, t) .
$$




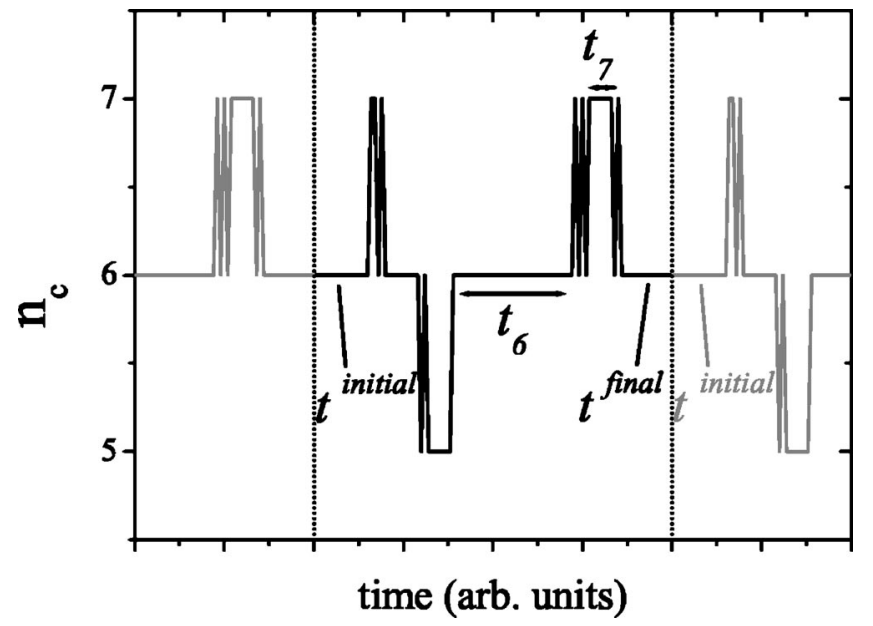

FIG. 2. A typical example of a single-particle coordination track: $n_{c}$ as a function of time (in black). Examples of time intervals for coordination numbers 6 and 7 are indicated as $t_{6}$ and $t_{7}$. The grey lines illustrate the looping of the tracks to properly take into account the initial and final time intervals (respectively indicated as $t^{\text {initial }}$ and $\left.t^{\text {final }}\right)$, as described in the text.

\section{Topological lifetimes}

As a measure for the time scale corresponding to local structural relaxation, we introduce the so-called topological lifetime. This topological lifetime of a given coordination number, $\tau_{n_{c}}$, is defined as the average time that a particle keeps the same coordination number between two $n_{c}$ changes. To calculate the lifetime, we followed the behavior of $n_{c}$ as a function of time for the single particles. Figure 2 shows a typical example of such a single-particle coordination track. These coordination tracks allow us to measure the time intervals $t_{n_{c}}$ that a particle retains the same coordination number. An example of such a time interval $t_{n_{c}}$ for $n_{c}=6$ and 7 is indicated in Fig. 2. Thus, all the single-particle coordination tracks (from all the tracked particles) provide a whole set of time intervals $t_{n_{c}}$ for each coordination number $n_{c}$. The lifetime of a given coordination number is then defined as the average over that whole set of time intervals:

$$
\tau_{n_{c}}=\frac{1}{M_{n_{c}}} \sum_{i=1}^{M_{n_{c}}} t_{n_{c}}^{(i)}
$$

where $M_{n_{c}}$ is thus the total number of time intervals $t_{n_{c}}$ in all single-particle coordination tracks.

Due to the finite length of a coordination track, the initial and final time intervals ( $t^{\text {initial }}$ and $t^{\text {final }}$ in Fig. 2) are not properly bounded by two $n_{c}$ changes. To correct for this, we loop the coordination tracks as illustated in Fig. 2. Now, there are three different cases possible. First, if the initial coordination number is equal to the final coordination number (as in Fig. 2), a time interval equal to the sum of $t^{\text {initial }}$ and $t^{\text {final }}$ is taken into account. Second, if the initial coordination number is not equal to the final coordination number, just $t^{\text {initial }}$ and $t^{\text {final }}$ are taken as such. And finally, if $n_{c}$ is constant throughout the whole coordination track, a time in- terval equal to the length of the coordination track is taken into account.

To improve statistics, we demand the minimum coordination track length (i.e., the minimum number of frames that a particle is tracked) to be $20 \%$ of the total time series. Increasing this length mainly affects the statistics of the defective particles, as they move faster and are more difficult to track. On the other hand, a smaller minimum track length enhances the effects of the finite track length. The maximum track length is per definition equal to the full time series. This limits the maximum lifetime to the total of the time series, thereby underestimating the very large lifetimes. Although the obtained lifetimes are slightly influenced by values of the minimum and maximum track length, the results do not change significantly.

Here and below, all distances are given in units of the particle diameter and time is represented in units of the Brownian time $\tau_{b}$, being the required time for a particle to diffuse over its own diameter at infinite dilution. Here, $\tau_{b}$ $=d^{2} / 6 D_{0}=1.6092 s$, where $D_{0}$ is the diffusion constant at infinite dilution.

\section{RESULTS}

\section{A. Structure}

Due to the slight polydispersity, crystallization was virtually absent in the bulk which allowed detailed study of glassy dynamics [40]. The situation is completely different at the bottom glass wall of the sample container. Here the system shows significant ordering [7], which may be explained by the enormous increase of the crystal nucleation rate in the vicinity of a wall [10]. Furthermore, size fractionation has been suggested to suppress the local degree of polydispersity, which could enhance crystallization as well [50]. Upon increasing the volume fraction the system shows a reentrant melting transition $(\phi>0.57)[7]$. Using the formalisms of the Kosterlitz-Thouless-Halperin-Nelson-Young theory for 2D melting [23-25], it turned out that the ordered phase $(0.52$ $\leqslant \phi \leqslant 0.57$ ) has a hexatic structure rather than crystalline [7]. Thus, the structural sequence of the first layer in our system for increasing volume fraction is, respectively, fluid, hexatic, and reentrant fluid [7].

The defect structure corroborates the hexatic structure. Figure 3 shows Delaunay triangulations of the system at $\phi$ $=0.55$ (hexatic), 0.57 (hexatic), and $\phi=0.60$ (remelting). The defects are color coded (see the caption of Fig. 3). In the hexatic regions we indeed observe free dislocations (5-7 disclination pairs) as well as somewhat larger compact defect clusters. These clusters have a typical size of three to four particle diameters consistent with earlier reports [5]. At a volume fraction of 0.55 clearly a grain boundary, a more-orless closed loop of defects, is observed. The lattice orientation within differs significantly from the orientation outside the grain boundary, which destroys the orientational order [5]. However, away from the grain boundary, the defect structure is similar to the one observed at $\phi=0.57$. At $\phi$ $=0.60$, where the system is remelting, the concentration of defects has increased significantly. The defects now percolate 

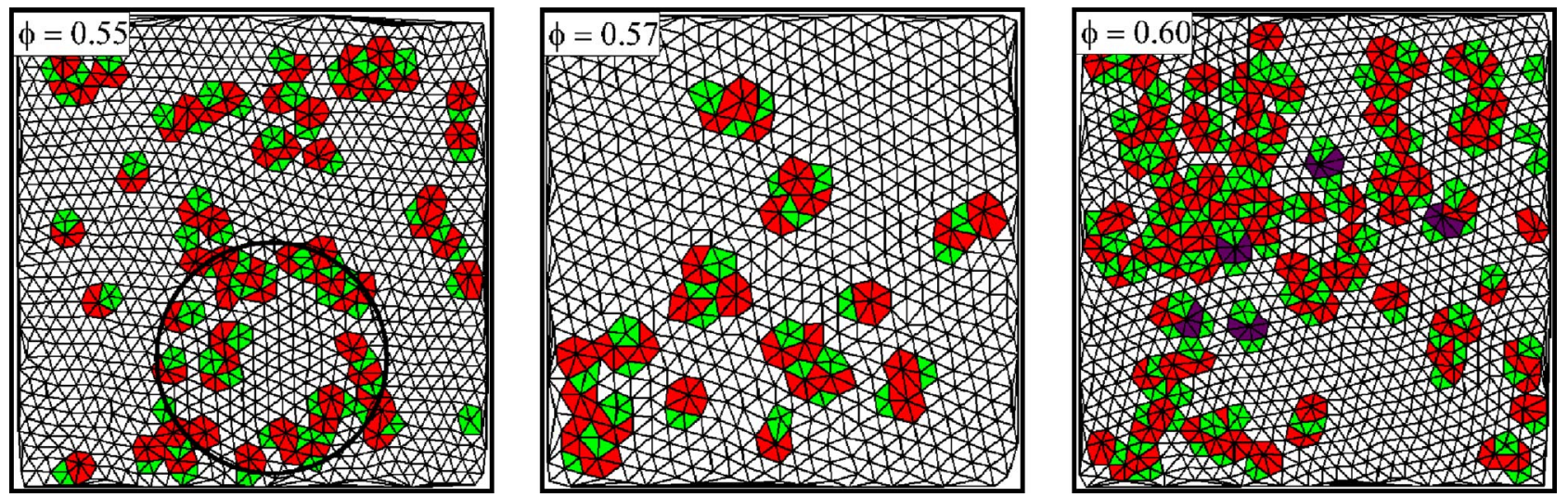

FIG. 3. (color online) Delaunay triangulations of the system at different volume fractions. From left to right: $\phi=0.55$ (hexatic, 50 $\times 50 \mu \mathrm{m}^{2}$ ), 0.57 (hexatic, $40 \times 40 \mu \mathrm{m}^{2}$ ), and 0.60 (remelting, $50 \times 50 \mu \mathrm{m}^{2}$ ). At $\phi=0.55$ the grain boundary is indicated by the black circle. The color code for the defects is as follows: fourfold, blue; fivefold, green; sixfold, no color; sevenfold, red; eightfold, purple.

through the whole image, and we also observe free disclinations.

The defect structure can be quantified by calculating the fraction of $n_{c}$-coordinated particles [Eq. (1)] as a function of volume fraction, which is shown in Fig. 4. The reentrant character is reflected by the maximum (minimum) in $X_{6}$ $\left(X_{5}, X_{7}\right)$ at $0.52 \leqslant \phi \leqslant 0.57$, which corresponds to the hexatic region. More interesting, $X_{5} \cong X_{7}$ in this region, indicating that the disclinations are indeed bound in pairs (dislocations). Above and below the hexatic region these fractions are not equal anymore, pointing towards the generation of free disclinations. Furthermore, we observe at all volume fractions that there are hardly particles having a coordination number different from 5, 6, or 7. As a consequence, the system is fairly well described by taking into account only the particles having $n_{c} \in[5,7]$.

The presence of a hexatic structure might reflect the twodimensional character of the first layer at the wall, as hexatic phases mainly have been observed in monodisperse [5,26-28] as well as polydisperse 2D colloidal systems [51-54]. The strong layering of a 3D fluid at a single wall [3] corroborates the $2 \mathrm{D}$ character of the first layer at a wall,

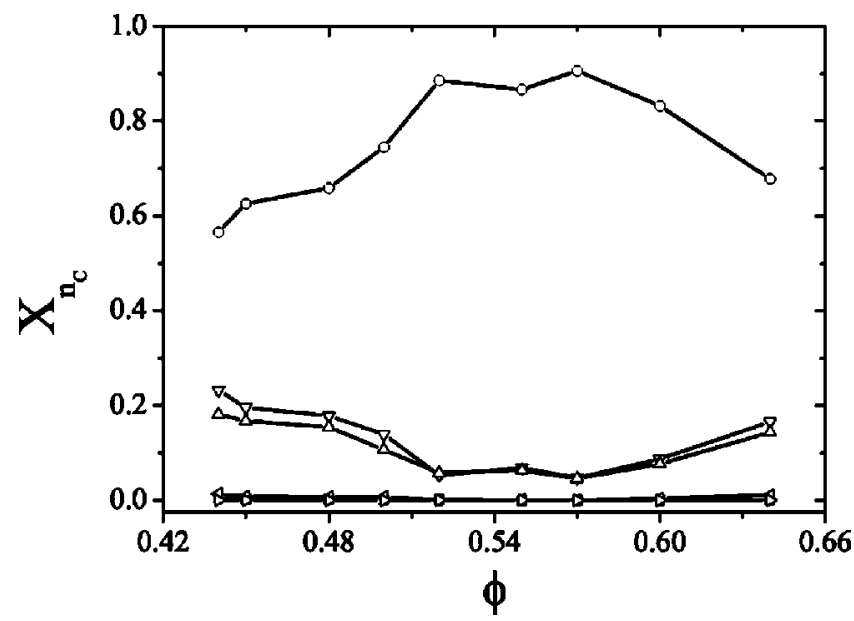

FIG. 4. Fraction of defects as a function of volume fraction. Symbols: $n_{c}=4(\square), 5(\nabla), 6(\bigcirc), 7(\triangle), 8(\triangleleft)$, and $9(\triangleright)$. although structural and dynamical differences between 2D and the first 3D layer have been reported $[4,12]$. However, in monodisperse 3D systems at a wall hexatic structures have not been reported $[3,6,8-11,55]$. Therefore, the particle polydispersity might play a crucial role in the formation of the hexatic structure in the first 3D layer in our system as geometrical frustration is known to promote the formation of a hexaticlike structure [56-58].

\section{B. Dynamics}

The reentrant melting transition is accompanied by an increase in the mean-squared displacement (Fig. 5), ruling out the possibility of glass. Note that the displacements are small compared to the particle diameter ( interlayer distance), so interlayer hopping is expected to be insignificant on the time scale of the experiment. Therefore, hopping between the first and second layer is hardly observed. The increase of the

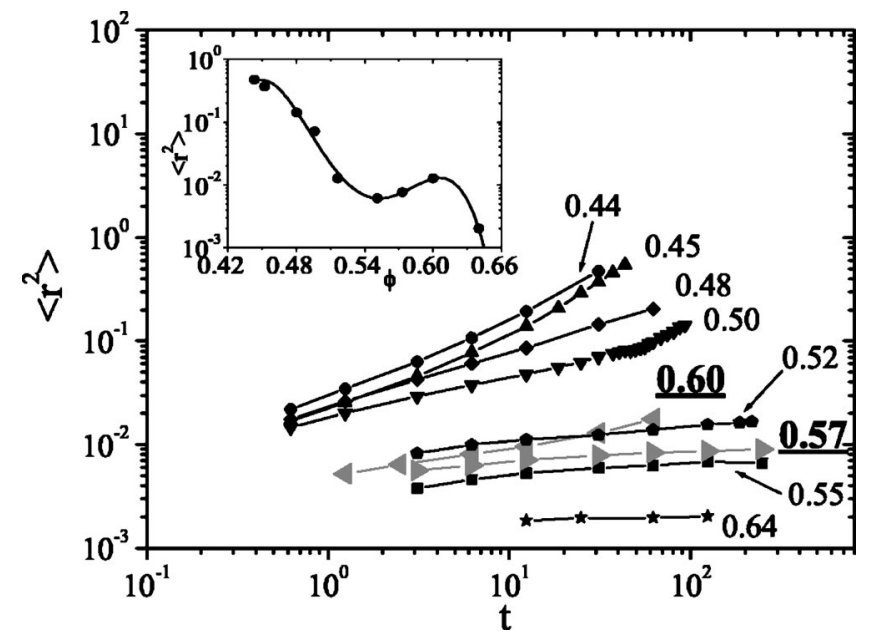

FIG. 5. Mean-squared displacements as a function of time $t$ for different volume fractions. For $\phi=0.57$ and $\phi=0.60$ (grey) the $\left\langle r^{2}\right\rangle$ clearly increases. Note that this increase is present for all studied times $t$. The inset is a vertical cut through the $\left(r^{2}\right.$ vs $\left.t\right)$ plot and shows $\left\langle r^{2}\right\rangle$ as a function of $\phi$ for $t=31.07$ Brownian times. The line in the inset is to guide the eye. 

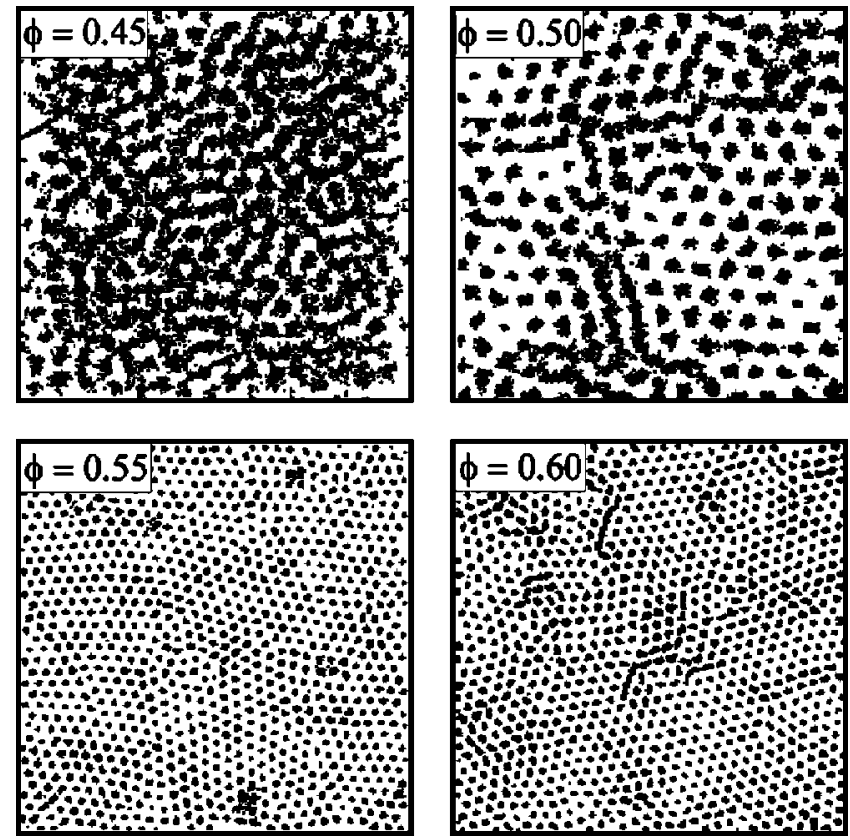

FIG. 6. Particle positions over 100 images (time steps) for several volume fractions: $\phi=0.45$ (fluid, $23 \times 23 \mu \mathrm{m}^{2}$ ), 0.50 (at freezing, $30 \times 30 \mu \mathrm{m}^{2}$ ), 0.55 (hexatic, $50 \times 50 \mu \mathrm{m}^{2}$ ), and 0.60 (remelting, $\left.50 \times 50 \mu \mathrm{m}^{2}\right)$.

mobility is expected to be correlated to the defect structure of the system. To look into this, we show the positions of the centers of the particles over 100 time steps for several volume fractions (Fig. 6). In the isotropic fluid at $\phi=0.45$ the particles exhibit homogeneous dynamics as expected for a liquid. At freezing, $\phi=0.50$, we clearly observe ordered and disordered regions, as well as more and less mobile particles. The more mobile particles exhibit typical stringlike, cooperative motion [42]. Furthermore, the mobile particles tend to be located in the more disordered, defective regions $[5,6,30,32,34-36,43]$. At a volume fraction of 0.55 (hexaticlike structure), the system seems to be dynamically homogeneous. Even the particles in the defective regions-for example, in the grain boundary as shown in Fig. 3-do not show significant higher mobility. As the system starts to remelt at $\phi=0.60$, the ordered hexatic structure starts to break up by introducing more defects (Fig. 3). Similar to the situation at $\phi=0.50$, the more mobile particles are typically located in the more defective regions. Furthermore, the stringlike, cooperative motion, characteristic of the presence of dynamical heterogeneities, is observed again. These dynamical heterogeneities manifest themselves also as non-Gaussian contributions to the self-part of the Van Hove correlation function [Eq. (7)]. If the system is dynamical homogeneous, $G_{s}$ is expected to have a single-Gaussian shape [59]. To illustrate that this is indeed the case, we show in Fig. 7 the self-part of the Van Hove correlation function for the isotropic liquid at $\phi=0.45$ and for the dynamical heterogeneous remelting system at $\phi=0.60$. The $G_{s}$ for the fluid is well described by a single-Gaussian distribution, whereas the $G_{s}$ at $\phi=0.60$ shows significant deviations from a singleGaussian distribution. Moreover, it can be fairly well described by a sum of two Gaussians, pointing towards the
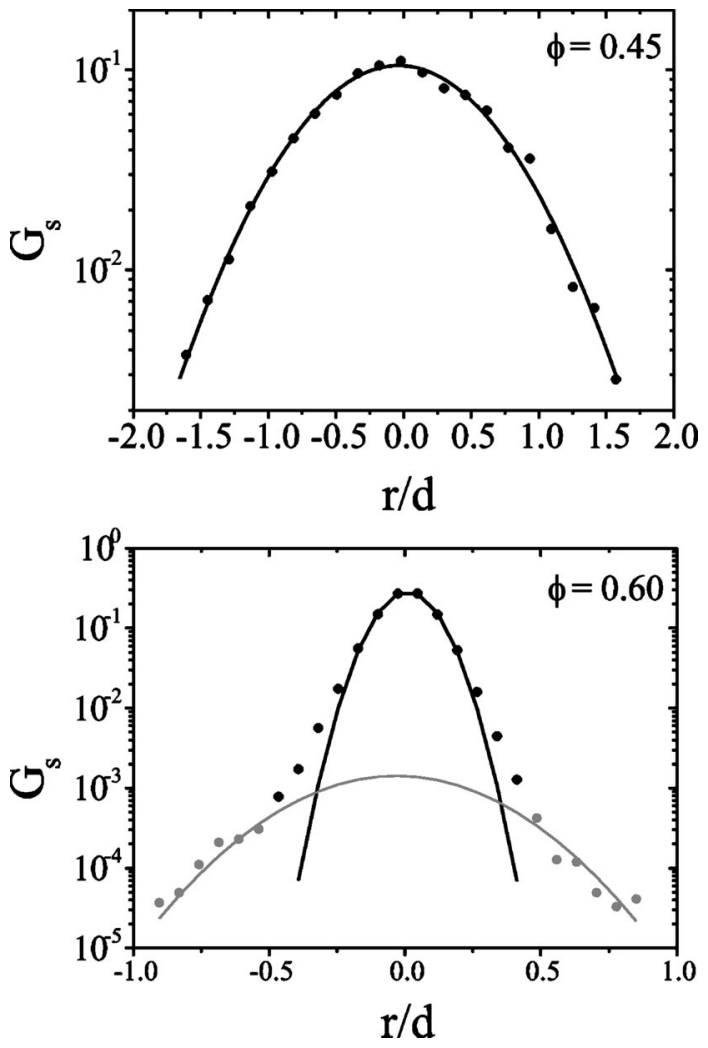

FIG. 7. Self-part of the Van Hove correlation function $G_{s}$. Top: in the isotropic liquid $(\phi=0.45)$ the $G_{s}$ is a single-Gaussian function, which indicates diffusive motion. At $\phi=0.60, G_{s}$ strongly deviates from a single-Gaussian function, indicating the presence of dynamical heterogeneities. Moreover, the $G_{S}$ is fairly well described by a sum of two Gaussians: a wide one (grey line) for the fast subset (grey dots) and a narrow one (black line) for the slow subset (black dots).

existence of a slow (the wide Gaussian) and a fast (the narrow Gaussian) subset of particles.

\section{Structure, dynamics, and topological lifetimes}

Although the correlation between structure and dynamics seems to be well accepted on a qualitative level, it is not always obvious. For example, our system at $\phi=0.55$ is dynamically fairly homogeneous, while structurally clearly not, as there are clearly ordered and disordered regions (recall the presence of a grain boundary) present. Futhermore, in bulk, our system is structurally homogeneous (disordered), but still exhibits dynamical heterogeneities [40]. In addition, the present tools are unable to quantitatively link statics and dynamics. To gain more quantitative insight into the relation between structure and dynamics, we studied the fluctuations in the particle topology in terms of topological lifetimes as defined in Sec. III C. The lifetime is a measure for the stability of a local structure characterized by $n_{c}$ and directly correlates local structure and dynamics.

As a start, we show that our system obeys ergodicity, proving the meaningfulness of our definition of topological lifetimes. The defect statistics can be expressed in terms of lifetimes demanding ergodicity; i.e., the ensemble average, 


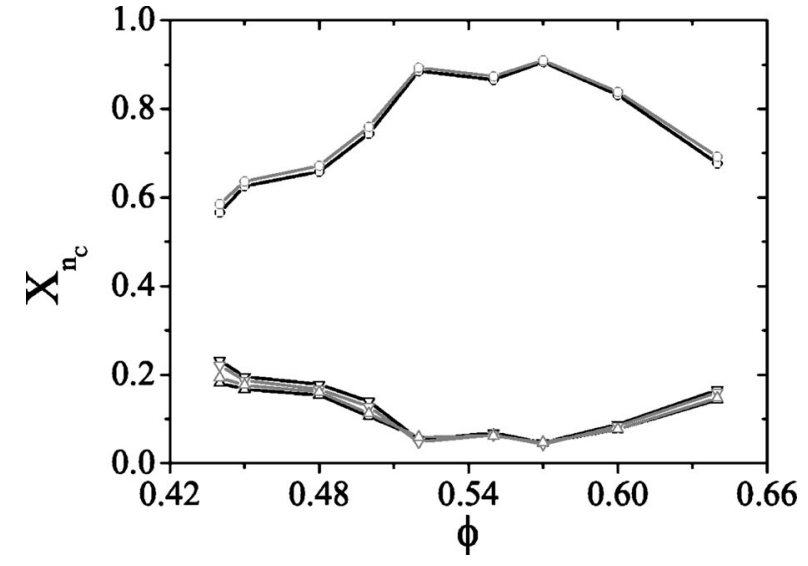

FIG. 8. Fraction of defects as a function of volume fraction: ensemble average [Eq. (1), in black] and time average [Eq. (5), in grey]. Symbols: $n_{c}=5(\nabla), 6(\bigcirc)$, and $7(\triangle)$.

as defined by Eq. (1), must be equal to the time average:

$$
X_{n_{c}}=\frac{N_{n_{c}}(\text { ens av) }}{N}=\frac{\tau_{n_{c}} M_{n_{c}}}{\sum_{n_{c}} \tau_{n_{c}} M_{n_{c}}}{ }^{\text {(time av) }} .
$$

In Fig. 8 the results of Eq. (5) are presented for $n_{c} \in[5,7]$. It is observed that the defect statistics obtained via the time average very accurately reproduces the result from the ensemble average, thereby demonstrating the ergodicity of our system and the meaningfulness of the lifetimes.

Next, we investigate the coordination number dependence of the lifetimes for different volume fractions. This is shown in Fig. 9. It is clearly observed that for all volume fractions the lifetime for $n_{c}=6$ is always the largest. Fivefold- and sevenfold-coordinated particles have comparable lifetimes, but much smaller than the sixfold-coordinated particles. This implies that local structures involving sixfold coordination are always the most stable. This is a direct consequence of the average coordination number in $2 \mathrm{D}$ liquids being 6 [5]. As a result, at all volume fractions the defective particles have the tendency to return to a sixfold-coordinated state. This is reflected by the defect statistics (Fig. 4), but can be

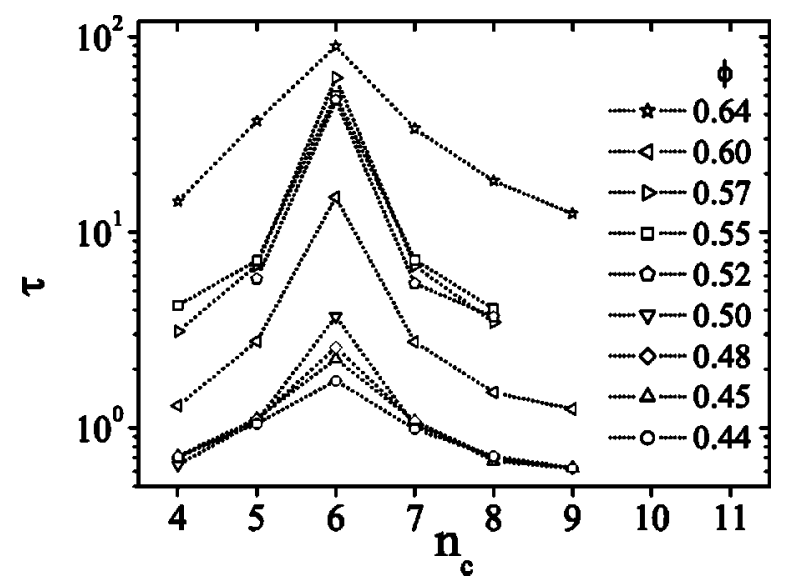

FIG. 9. The lifetime $\tau$ in units of $\tau_{b}$ as a function of the coordination number $n_{c}$ for different volume fractions.

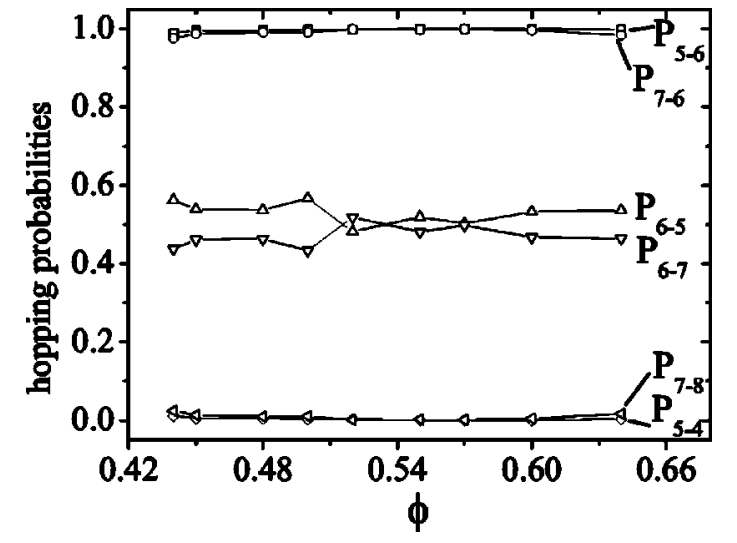

FIG. 10. Hopping probabilities as obtained from Eq. (6).

shown more directly by defining a "hopping probability"that is, the probability that a particle with a given coordination number $n_{c}$ hops to $n_{c} \pm 1$ :

$$
P_{\left(n_{c} \rightarrow n_{c} \pm 1\right)}=\frac{X_{\left(n_{c} \pm 1\right)}}{X_{\left(n_{c}-1\right)}+X_{\left(n_{c}+1\right)}},
$$

where we assume that $\Delta n_{c} \equiv \pm 1$. Here, the hopping probability is expressed in terms of defect fractions. Hence, it can also be expressed in terms of lifetimes, as the system is ergodic [Eq. (5) is valid]. The hopping probability as a function of volume fraction is presented in Fig. 10. The probability that a particle goes back to $n_{c}=6$ is indeed observed to be close to 1 for all volume fractions-i.e., regardless of the structure of the system. Consistently, the probability that a fivefold- or sevenfold-coordinated particle becomes, respectively, a fourfold- or eightfold-coordinated particle is negligible. Note that for volume fractions outside the ordered (hexaticlike) region $(0.52 \leqslant \phi \leqslant 0.57)$ a sixfold particle is more likely to become fivefold coordinated rather than sevenfold $\left(P_{(6 \rightarrow 5)}>P_{(6 \rightarrow 7)}\right)$, whereas within the hexatic region, these probabilities are almost equal.

Furthermore, the lifetimes also exhibit a strong volume fraction dependence for each distinct coordination number (Fig. 9). For all $n_{c}$ the lifetimes at $\phi=0.64$ (close to random close packing) are the largest. If we go down along the lifetime axis, we successively encounter the three branches that correspond to the hexaticlike region $(0.52 \leqslant \phi \leqslant 0.57)$, the reentrant fluid branch at $\phi=0.60$, and finally the fluid regime $(\phi \leqslant 0.50)$. We should mention that the lifetimes for the rare coordination numbers-i.e., $n_{c}=4,8$, and 9-are not very reliable because of very poor statistics as the corresponding $X_{4}, X_{8}$, and $X_{9}$ are just very small. The $\phi$ dependence of the lifetimes for $n_{c} \in[5,7]$ is shown more clearly in Fig. 11 . Here, the different structural regimes (fluid, hexatic, remelting fluid, and close to random close packing) as already identified from Fig. 9 are observed even better. This enables us to estimate a characteristic lifetime for $n_{c}=6$ in each structural region (as a function of $\phi$ ). In the fluid region $\tau_{6} \simeq 3 \tau_{b}$, in the hexatic region $\tau_{6} \simeq 55 \tau_{b}$, in the reentrant fluid $\tau_{6} \simeq 15 \tau_{b}$, and close to random close packing $\tau_{6} \simeq 80 \tau_{b}$. Our values for the fluid and hexatic regions are in reasonable agreement with 


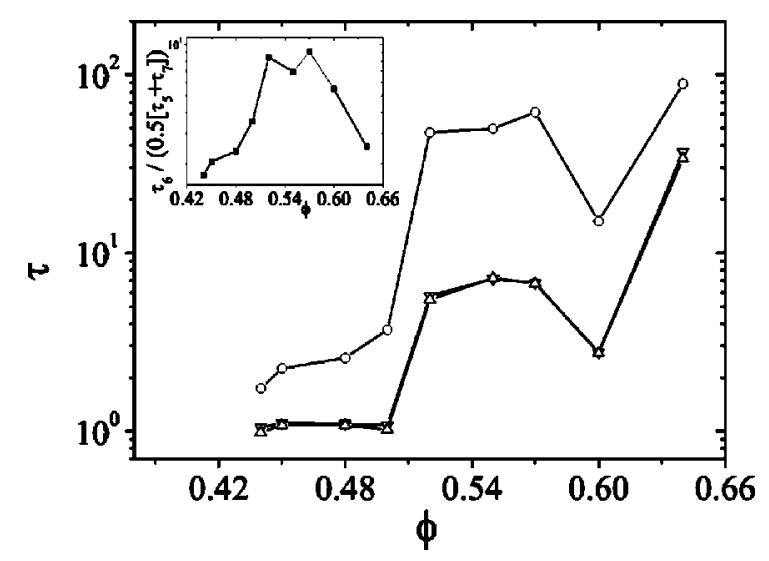

FIG. 11. Lifetime as a function of volume fraction for different coordination numbers. Symbols: $n_{c}=5(\nabla), 6(\bigcirc)$, and $7(\triangle)$. The inset shows the ratio $\tau_{6} /\left(0.5\left[\tau_{5}+\tau_{7}\right]\right)$ as a function of the volume fraction.

the orientational correlation time as defined by Murray et al. $\left[-d \ln g_{6}^{\prime}(0, t) / d t\right]^{-1}[4,5,37]$, which is a measure of the time decay of the bond-orientational order [5]. This agreement is expected as the bond-orientational order is highly sensitive to the coordination number and, in particular, to the bond angles of the nearest neighbors. As long as a particle stays sixfold coordinated, the sixfold bond-orientational order is preserved to some extent (there might be fluctuations in bond angles). If the $n_{c}$ changes, the bond-orientational order is destroyed, indicating that our $\tau_{6}$ is similar to the orientational correlation time defined by Murray [5].

In addition, we can also determine these time scales for different local structures-i.e., different coordination numbers-at all volume fractions. For example, for $n_{c}=5$ these characteristic lifetimes are, respectively, in the fluid $\tau_{5} \simeq \tau_{b}$, in the hexatic $\tau_{5} \simeq 7 \tau_{b}$, in the reentrant fluid $\tau_{5}$ $\simeq 3 \tau_{b}$, and close to random close packing $\tau_{5} \simeq 35 \tau_{b}$. Note that the $\tau_{7}$ is almost equal to $\tau_{5}$. We further observe in Fig. 11 that $\tau_{5}\left(\tau_{7}\right)$ exhibits a similar volume fraction dependence as $\tau_{6}$. Again, the distinct structural regions are identified. However, the magnitude of $\tau_{5}\left(\tau_{7}\right)$ does not scale with $\phi$ as $\tau_{6}$ does, which is evident from the inset of Fig. 11. The inset shows the ratio between $\tau_{6}$ and the average of $\tau_{5}$ and $\tau_{7}$ $\left(\tau_{6} /\left(0.5\left[\tau_{5}+\tau_{7}\right]\right)\right)$ as a function of volume fraction. In the fluid phase and close to random close packing, this ratio is roughly 2 , in the hexatic region about 8 and in the reentrant fluid approximately 5 . Thus, upon ordering at $\phi \simeq 0.50$, the difference between the lifetimes corresponding to, respectively, sixfold-coordinated (ordered) and non-sixfoldcoordinated (disordered) particles increases. Therefore, the ratio $\tau_{6} /\left(0.5\left[\tau_{5}+\tau_{7}\right]\right)$ as well as $\tau_{5}, \tau_{6}, \tau_{7}$ identify the different structural regions and might therefore be identified as a dynamical order parameters for ordering $[37,38]$.

Besides the characteristic lifetimes for each structural region, we clearly recognize in Fig. 11 the global behavior of the mean-squared displacement as function of $\phi$ as shown in the inset of Fig. 5. Interestingly, this suggests that there is a quantitative correlation between topological lifetimes and mobility. Hence, the lifetimes are more or less determined by the local mobility of the particles. If a given particle and/or

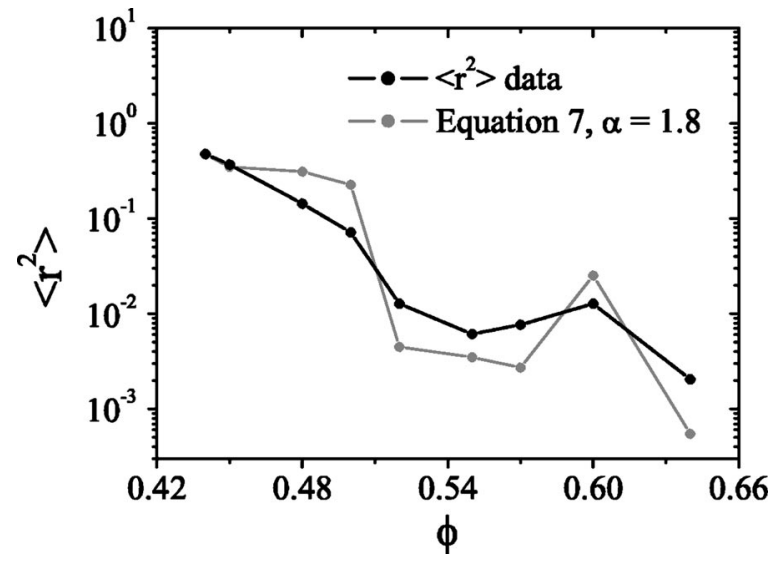

FIG. 12. Mean-squared displacement as a function of volume fraction for $t=31.07$ Brownian times. The experimental data in are black and the results obtained using Eq. (7) are in grey.

(one of) its neighbors is moving significantly, the coordination number of the given particle changes, thereby setting a typical lifetime. Therefore, a very large lifetime indicates that the local structure of a particle does not change, corresponding to a low mobility in that particular region. In other words, if the lifetime is large, the mobility is small; hence, the mean-squared displacement is expected to scale with the inverse of the lifetime (which can be interpreted as a hopping frequency). The weight of the contribution of $\tau_{n_{c}}$ to the mobility (mean-squared displacement) is given by the concentration of particles being $n_{c}$ coordinated, $X_{n_{c}}$. Finally, we have to sum over all coordination numbers to take into account all the particles. Although the qualitative trend is described correctly (data not shown), we may improve the quantitative agreement. Now, the lifetimes are taken into account as a single-particle property. However, if the coordination number of given particle changes (setting the lifetime), in the dense system the coordination number of a neighboring particle changes as well. Therefore, correlations could be important and the lifetime may have a larger influence on the mean-squared displacement. To account for this and improve the quantitative agreement one might introduce an additional parameter $\alpha$ such that rather than $\left\langle r^{2}\right\rangle \propto 1 / \tau_{n_{c}}$, the meansquared displacement may scale as

$$
\left\langle r^{2}(\phi)\right\rangle \propto \sum_{n_{c}} \frac{1}{\tau_{n_{c}}^{\alpha}(\phi)} X_{n_{c}}(\phi) .
$$

By fitting Eq. (7) to the experimental data a value of $\alpha$ $=1.8$ was obtained. In Fig. 12 the result of Eq. (7) with $\alpha$ $=1.8$ and the experimental data of the inset of Fig. 5 are shown. Although it remains unclear what exactly determines the value of $\alpha$, we clearly observe that the trend of the data is described fairly well by Eq. (7). This implies that particles undergoing a coordination-number change dominate the behavior of the mean-squared displacement, which confirms that defective particles (either before or after changing $n_{c}$ : $\left.n_{c} \neq 6\right)$ are the most mobile particles.

\section{CONCLUSION}

We have quantitatively analyzed the correlation between structure and dynamics on a single-particle level in terms of 
topological lifetime $\tau$, being the average time that a particle spends having the same coordination number. Our analysis is easily applicable to other systems such as confined quasi (2D) systems and bulk (3D) systems. The defective (nonsixfold-coordinated) particles exhibit shorter lifetimes than sixfold-coordinated particles, indicating that the mobility of the system is larger in defective regions. The lifetime itself shows a strong volume fraction dependence. In particular, the global behavior of the mean-squared displacement is proportional to the inverse of the lifetime (hopping frequency), showing that particles changing their coordination number contribute most to the mobility.

\section{ACKNOWLEDGMENTS}

We thank Alfons van Blaaderen for help during the experiments and useful discussions and Dirk Aarts and Andrei Petukhov for helpful discussions. Gilles Bosma is thanked for the particle synthesis. This work is part of the research programme of the Stichting voor Fundamenteel Onderzoek der Materie (FOM), financially supported by the Nederlandse Organisatie voor Wetenschappelijk Onderzoek (NWO).
[1] S. Dietrich, Phase Transitions and Critical Phenomena (Academic, London, 1988).

[2] C. Bechinger, Curr. Opin. Colloid Interface Sci. 7, 204 (2002).

[3] D. H. Van Winkle and C. A. Murray, J. Chem. Phys. 89, 3385 (1988).

[4] C. A. Murray, W. O. Sprenger, and R. A. Wenk, Phys. Rev. B 42, 688 (1990).

[5] C. A. Murray, in Bond-Orientational Order in Condensed Matter Systems, edited by K. Strandburg, (Springer-Verlag, New York, 1992), pp. 137-215.

[6] J. P. Hoogenboom, P. Vergeer, and A. van Blaaderen, J. Chem. Phys. 119, 3371 (2003).

[7] R. P. A. Dullens and W. K. Kegel, Phys. Rev. Lett. 92, 195702 (2004).

[8] D. J. Courtemanche and F. van Swol, Phys. Rev. Lett. 69, 2078 (1992).

[9] D. J. Courtemanche, T. A. Pasmore, and F. van Swol, Mol. Phys. 80, 861 (1993).

[10] S. Auer and D. Frenkel, Phys. Rev. Lett. 91, 015703 (2003).

[11] M. Dijkstra, Phys. Rev. Lett. 93, 108303 (2004).

[12] C. A. Murray, W. O. Sprenger, and R. A. Wenk, J. Phys.: Condens. Matter 2, A385 (1990).

[13] D. G. Grier and C. A. Murray, J. Chem. Phys. 100, 9088 (1994).

[14] M. Schmidt and H. Löwen, Phys. Rev. Lett. 76, 4552 (1996).

[15] A. Cacciuto, S. Auer, and D. Frenkel, Nature (London) 428, 404 (2004).

[16] A. Kose and S. Hachisu, J. Colloid Interface Sci. 55, 487 (1976).

[17] A. Gast, W. Russel, and C. Hall, J. Colloid Interface Sci. 55, 161 (1986).

[18] P. D. Kaplan, J. L. Rouke, A. G. Yodh, and D. J. Pine, Phys. Rev. Lett. 72, 582 (1994).

[19] A. Dinsmore, P. Warren, W. Poon, and A. Yodh, Europhys. Lett. 40, 337 (1997).

[20] A. van Blaaderen and P. Wiltzius, Nature (London) 385, 321 (1997).

[21] M. Heni and H. Löwen, Phys. Rev. Lett. 85, 3668 (2000).

[22] Y. Tang, R. M. Malzbender, R. C. Mockler, W. J. O’sullivan, and J. A. Beall, J. Phys. A 20, L189 (1987).

[23] J. M. Kosterlitz and D. J. Thouless, J. Phys. C 6, 1181 (1973).

[24] B. I. Halperin and D. R. Nelson, Phys. Rev. Lett. 41, 121 (1978).
[25] A. P. Young, Phys. Rev. B 19, 1855 (1979).

[26] R. E. Kusner, J. A. Mann, J. Kerins, and A. J. Dahm, Phys. Rev. Lett. 73, 3113 (1994).

[27] K. Zahn, R. Lenke, and G. Maret, Phys. Rev. Lett. 82, 2721 (1999).

[28] A. H. Marcus and S. A. Rice, Phys. Rev. Lett. 77, 2577 (1996).

[29] C. A. Murray and D. H. Van Winkle, Phys. Rev. Lett. 58, 1200 (1987).

[30] Y. Tang, A. J. Armstrong, R. C. Mockler, and W. J. O'Sullivan, Phys. Rev. Lett. 62, 2401 (1989).

[31] A. H. Marcus, J. Schofield, and S. A. Rice, Phys. Rev. E 60, 5725 (1999).

[32] B. Cui, B. Lin, and S. A. Rice, J. Chem. Phys. 114, 9142 (2001).

[33] D. N. Perera and P. Harrowell, J. Chem. Phys. 111, 5441 (1999).

[34] C. Reichhardt and C. J. Olson Reichhardt, Phys. Rev. Lett. 90, 095504 (2003).

[35] R. Zangi and S. A. Rice, Phys. Rev. E 68, 061508 (2003).

[36] R. Zangi and S. A. Rice, Phys. Rev. Lett. 92, 035502 (2004).

[37] C. A. Murray and R. A. Wenk, Phys. Rev. Lett. 62, 1643 (1989).

[38] K. Zahn and G. Maret, Phys. Rev. Lett. 85, 3656 (2000).

[39] A. Pertsinidis and X. S. Ling, Nature (London) 413, 147 (2001).

[40] W. K. Kegel and A. van Blaaderen, Science 287, 290 (2000).

[41] E. R. Weeks, J. C. Crocker, A. C. Levitt, A. Schofield, and D. A. Weitz, Science 287, 627 (2000).

[42] S. C. Glotzer, J. Non-Cryst. Solids 274, 342 (2000).

[43] E. R. Weeks and D. A. Weitz, Phys. Rev. Lett. 89, 095704 (2002).

[44] A. van Blaaderen and A. Vrij, Langmuir 8, 2921 (1992).

[45] G. Bosma, Z. Dogic, C. Pathmamanoharan, W. K. Kegel, and A. van Blaaderen (unpublished).

[46] L. Antl, J. W. Goodwin, R. D. Hill, S. M. Owens, and S. Papworth, Colloids Surf. 17, 67 (1986).

[47] E. H. A. de Hoog, W. K. Kegel, A. van Blaaderen, and H. N. W. Lekkerkerker, Phys. Rev. E 64, 021407 (2001).

[48] W. Schaertl and H. Sillescu, J. Stat. Phys. 77, 1007 (1994).

[49] J. C. Crocker and D. G. Grier, J. Colloid Interface Sci. 179, 298 (1996).

[50] M. Buzzacchi, I. Pagonabarraga, and N. B. Wilding, J. Chem. 
Phys. 121, 11362 (2004).

[51] M. R. Sadr-Lahijany, P. Ray, and H. E. Stanley, Phys. Rev. Lett. 79, 3206 (1997).

[52] L. Santen and W. Krauth, e-print cond-mat/0107459.

[53] S. Pronk and D. Frenkel, Phys. Rev. E 69, 066123 (2004).

[54] J. J. Gray, D. H. Klein, B. A. Korgel, and R. T. Bonnecaze, Langmuir 17, 2317 (2001).

[55] J. E. Hug, F. van Swol, and C. F. Zukoski, Langmuir 11, 111 (1995).
[56] A. V. Petukhov, D. van der Beek, R. P. A. Dullens, I. P. Dolbnya, G. J. Vroege and H. N. W. Lekkerkerker (unpublished).

[57] D. R. Nelson and M. Rubinstein, Philos. Mag. A 46, 105 (1982).

[58] D. R. Nelson, Defects and Geometry in Condensed Matter Physics (Cambridge University Press, Cambridge, England, 2002).

[59] J. P. Hansen and I. R. McDonald, Theory of Simple Liquids, 2nd ed. (Academic Press, London, 1986). 